\title{
Short Term Electric Load Forecasting of 132/33KV Maiduguri Transmission Substation using Adaptive Neuro-Fuzzy Inference System (ANFIS)
}

\author{
Idakwo O. Harrison \\ Department of Engineering \\ University of Maiduguri \\ Teaching, Hospital.
}

\author{
Dan'isa, A. \\ Dept of Electrical Engineering \\ Bayero University Kano
}

\author{
Bello Ishaku \\ Department of Engineering \\ University of Maiduguri \\ Teaching, Hospital.
}

\begin{abstract}
This article provides a way of accurately predicting one-hourahead load of a utility company located in the North Eastern region of Nigeria based on Adaptive Neuro-Fuzzy Inference System (ANFIS). The inputs to the ANFIS are the next-hour temperature, next-hour humidity, day of the week, hour of the day, and the current-hour load. The output is the next-hour load of the entire system. All the data used span the period 2009 to 2012 (4 years). These parameters are non-linear, stochastic (random) and uncertain in nature. Adaptive Neurofuzzy based Inference System (ANFIS), an integrated system, comprising of fuzzy logic and Neural Network was used to model the next hour load, because it can address and solve problems related to non-linearity, randomness and uncertainty of data. $75 \%$ of the data was used for training and $25 \%$ for checking. From the analysis carried out on the ANFIS-based model; Mean absolute percentage error (MAPE) for a typical Monday, Wednesday and Friday was found to be $12.61 \%$, $12.76 \%$ and $12.12 \%$. The Mean absolute error (MAPE) on the entire test data was $24.76 \%$. The analysis shows satisfactory level of accuracy with regards to the ANFIS-based model developed in forecasting the next hour load especially with a correlation ( $r$ ) value of $84.64 \%$.
\end{abstract}

\section{General Terms}

Load Forecasting.

\section{Keywords}

ANFIS, Forecast, MAPE, APE

\section{INTRODUCTION}

Load forecasting in electric power company is the process of estimating active loads ahead of the actual load occurrence. It is an important component of electric power system to establish economical and reliable operations for power stations and their generating units. [1]. According to Chandra, et al, the estimation of future load demand is necessary because the forecast information can be used to aid optimal energy interchange between utilities, thereby saving valuable cost and also in managing the production and purchasing in an economically reasonable way [2]. It also plays a crucial role in helping the electric utility to make unit commitment decisions, to reduce the reserve capacity and to schedule device maintenance plan properly. It plays a key role in reducing the generation cost and also in improving the reliability of power systems. For short term operations and also for long term planning. The quality and accuracy of this forecast have big influence on the quality of the electrical distribution system planning. Electric load forecasting in power systems is a very important task for ensuring reliability and economical operation. The operation planning of electric systems as well as the maintenance and fuel reserve planning are all based on the load forecast results.
These days, electric load forecasting is a real-life problem in industry. The huge consumption of electric energy in these days has given the load forecasting a big attention from researchers and utility operators [3][4][5][6].Various traditional methods like time series method, regression-based methods have been used for the prediction of load [7]. The main drawback of these methods is the explicit relationship between different variables, requirement of heavy computational time and large amount of memory space. This leads to search for new methods which can solve the above limitations and give better result.

In this paper, Adaptive Neuro Fuzzy Inference system (ANFIS) was used to design a model that can find a solution to the short term load forecasting problem. Depending on the period of the forecast done, load forecasting can be classified into three different types [8].

- Short-term load forecasting, which forecasts within a time period of one week or less

- Medium-term load forecasting, which forecasts within a time period of one month to one year.

- Long-term load forecasting with a time period of more than one year.

Medium-term and long-term load forecasts play an important role in power system planning, and short-term load forecasting is critical for reliable and efficient operation of power systems. While medium-term and long-term load forecasts are mainly based on the prediction of the future economy status, growth rate of the population, the short-term load forecasting (STLF) is based largely on weather conditions [9].

\section{LITERATURE REVIEW}

\subsection{Load Forecasting}

The classification of load forecast model into short term, medium term and long term are dependent on some certain factors which are very much influential to the forecast model. The factors that are of great importance when considering short term load forecast are basically time (i.e. time of the day, day of the week and hour of the day), weather and customer categories.

The factors that are of paramount importance in medium and long term forecast includes customer class ( i.e. residential, commercial and industrial), historical load and weather data, economic as well as demographic data, appliances connected as well as their age [10].

\subsection{Load Forecasting Methods}

The methods employed in the forecasting of electric load can be divided into statistical method [13] and artificial intelligent 
method. Regressional method, exponential smoothing, similar day approach and time series methods are all statistical method of load forecasting. The drawback of the statistical methods is their limit ability to model the non linearity of load consumption. Artificial intelligence methods includes expert systems, neural network, fuzzy logic, neuro fuzzy e.t.c The artificial intelligent method have the advantage of being more flexible and can handle complexity and non linearity. Of all the aforementioned methods, more attention is being shifted towards the field of neural network, fuzzy logic and neuro fuzzy [12] because of their ability to handle the non linear relationships between load and the factors affecting it [14].

\subsection{Adaptive Neuro-Fuzzy Inference System}

ANFIS is one of the artificial intelligent methods that could be applied in electric load forecasting. It is a hybrid system that combines the human reasoning style of fuzzy logic and the connectionist/ learning style of neural network. It is an adaptive network. An adaptive network is a network structure consisting of a number of nodes connected by directional links [15]. Each node represents a process unit and the links specifying the relationship between the nodes. Some of the nodes in ANFIS are adaptive while others are fixed. The output of the adaptive nodes depends on some modifiable parameters relevant to the nodes. The learning rule specifies how these parameters should be updated to minimize a prescribed error that measures the difference between the actual output and a desired (target) output. In the general case, each node in an adaptive network may have a node weights or parameters associated with them. The main learning rule applied to adaptive network is the back propagation learning rule that may be combined with other learning mechanism to accelerate the convergence of the learning process. The shape of a fuzzy membership function depends on a set of parameters, and changing these parameters changes the shape

of the membership function. Hence, the nodes of an adaptive network are well suited to the representation of the fuzzy system membership functions, and an adaptive network type structure can be adapted to represent the fuzzy inference system [16]. Using any of the learning rules related to adaptive networks, the parameters associated with the membership functions will be automatically modified through the learning process to model closely the relation described by a set of known input/output pairs.

\subsection{ANFIS Structure}

The triangular, trapezoidal, generalized bell shaped, pi shaped, $\mathrm{z}$ shaped, s shaped, to mention but a few, are the various membership function that exist on the anfis graphic user interface. In the context of this paper work, the ANFIS used consists of three guassian membership functions on each input. Two steps are involved in the ANFIS processes which are known as the training and testing step respectively. During training, membership function parameters (membership function shapes) are modified in a manner that causes the desired input/output relationship to be learned. The training set is shown to the network many times (iterations or epochs), until converge is obtained (usually, a mean square error between output and target is minimized). During testing, the used data should not be seen during the training process.

\subsection{Review of Applications of ANFIS in Load Forecasting}

Bakirtziz, et al. applied Fuzzy Neural Network (FNN) based approach to forecast the load for the Greek Power System.
They made use of the hourly loads of 1992 supplied by the Public Power Corporation (PPC), as the training data sets, while all the days in 1993 excluding holidays and irregular days were used for forecasting. A total of 168 FNN's were used, one for each day type and hour of the day. The result obtained was concluded to be superior to ANN [17]..

Different from the method applied by Bakirtziz, et al, Papdakis et al, came up with a different mechanism to use FNN for STLF which involved building different FNN's for each day of the week in every season instead of building one FNN for each hour of each type of day. Using historical load data, a representative load curve was formulated. To obtain the predicted load curve, the load curve of the representative day was transformed to fit the forecasted extremes. Results obtained proved satisfactory [18].

The work by Dash, et al. proposed a different type of FNN, which took fuzzy membership values of the load and other weather variables as the inputs to the neural network, and the membership values of the predicted load as the outputs [19]. After the FNN produced the initial forecast, a fuzzy expert system generating the load correction was used to produce the final forecast. The forecast was performed using the data from a utility in Virginia, and promising results of average, peak, and hourly load forecasts were reported. Comparisons were made to show the effectiveness of the fuzzy correction.

Srinivasan, et al, proposed another FNN model for one-day ahead load forecasting, which used a neural network to model the relationship between the load and temperature, and a fuzzy expert system to post process the output of the neural network [20]. The proposed approach was tested using the load and temperature data provided by Singapore power pte Ltd. One and a half years of data were used for training, and half a year of data was used for forecasting. The proposed FNN model was compared with an existing multiple linear regressions based model and showed lower forecasting errors for all types of days including holidays.

\section{RESEARCH METHODOLOGY 3.1 Introduction}

Maiduguri, located in North Eastern Nigeria (latitude $11.85^{\circ}$ $\mathrm{N}$ and longitude $13.16^{0} \mathrm{E}$ ) has a total of three $11 \mathrm{kV}$ feeders and six $33 \mathrm{kV}$ feeders feeding within and outside Maiduguri town. The feeders are named below

\section{THREE 11KV FEEDERS \\ - Kukawa $11 \mathrm{kV}$ Feeder 1 \\ - Industrial $11 \mathrm{kV}$ Feeder 1 \\ - $\quad$ Sir Kashim 11 kV Feeder 1 \\ SIX 33KV FEEDERS \\ - $\quad$ Benesheik $33 \mathrm{kV}$ Feeder 1 \\ - $\quad$ University $33 \mathrm{kV}$ feeder 2 \\ - $\quad$ Monguno $33 \mathrm{kV}$ Feeder 3 \\ - $\quad$ Bama $33 \mathrm{kV}$ Feeder 4 \\ - $\quad$ Damasak $33 \mathrm{kV}$ Feeder 5 \\ - $\quad$ Maiduguri town $33 \mathrm{kV}$ Feeder 6}

In the context of this paper work the combined total hourly load data from feeder line 2 (University) and feeder line 6 (Maiduguri) which are the two $33 \mathrm{kV}$ feeders serving Maiduguri metropolitan council are to be considered. 


\subsection{Collection of Data}

The principal instrument used for load data collection is the daily summary log sheets of The Power Holding Company of Nigeria PHCN. The data on Maiduguri feeder lines 6 and University feeder line 2, (the two major feeders feeding Maiduguri Metropolitain area) for the periods of January 2009 to December 2012 were obtained from the log sheets kept in the undertaking offices of the Power Holding Company of Nigeria (PHCN). The data included the hourly load data transmitted within the Metropolitain. The hourly weather data (Temperature and relative humidity) for the periods of January 2009 to December 2012 for Maiduguri Metropolitain were also collected from the world weather online web portal (www.worldweatheronline.com/Maiduguri-weatherhistory/Borno/NG.aspx.).

\subsection{Presentation of Data}

The Adaptive Neuro-fuzzy based inference system (ANFIS) is applied to model the next hour load forecast for the $132 / 33 \mathrm{kv}$ Maiduguri substation using time of the day, day of the week, next hour temperature, next hour relative humidity, and current hour load as the input variables. A total of twenty six thousand two hundred and eighty (26280) hourly data for the years 2009, 2010 and 2011 is used as the training data while eight thousand eight hundred and eighty four (8784) hourly data for the year 2012 is used as testing data set. A matrix of $26280 \times 6$ is generated, with the row number matching the number of hours for the year's 2009, 2010 and 2011. The first five (5) columns represent the inputs to the anfis model i.e. (hour of the day, day of the week, temperature, humidity and current hour load respectively. The last column represents the output to the anfis model. Each day of the week and time of the day is assigned to the temperature humidity and current load accordingly. During training, membership function parameters (membership function shapes) are modified in a manner that causes the desired input/output relationship to be learned. The training set is shown to the network many times (iterations or epochs), until converge is obtained (usually, a mean square error between output and target is minimized). The final obtained matrix is then converted from a .mat format (a format usable by MATLAB) to a .dat format which is a format usable by ANFIS using the MATLAB code "dlmwrite ('filename.dat', [variables])" and then saved in a computer location to be later used for training purpose. The testing data set is used for model validation which is the process by which the input vectors from input/output data sets on which the FIS was not trained, are presented to the trained FIS model, to see how well the FIS model predicts the corresponding data set output values. The testing data set lets you check the generalization capability of the resulting fuzzy inference system.

\subsection{Design of the ANFIS-Based Model}

For the design of the ANFIS model, selections of the following listed parameters were made.

\section{- Membership function type}

- Number of membership function

- Learning algorithm

- $\quad$ Epoch size

- Data size.

- Number of input variables.
The listed procedures below was adopted at the ANFIS Graphical user Interface (GUI) for the design of the model.

- Obtaining training data

- $\quad$ Data sizing

- Data partitioning

- Loading data sets.

- The ANFIS modeling criterion was adopted to effectively tune the membership function so as to minimize the output error and maximize performance index. The ANFIS structure obtained by the aforementioned parameters choosen is shown in fig 1 .

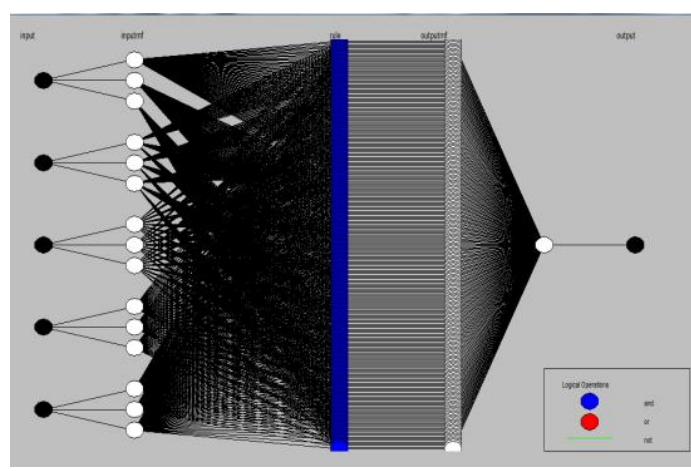

Fig 1: Structure of a 5 input ANFIS system

\subsection{Generating the Fuzzy Rules and Choosing the Membership Function}

After successfully loading the training data, the rules are generated by the grid partition method. Since there are five inputs with three membership functions, the rule set contains $3^{5}$, i.e., 243 rules. Grid partitioning is an approach for initializing the structure in a fuzzy inference system. In this method it generates rules by enumerating all possible combinations of membership functions of all inputs.

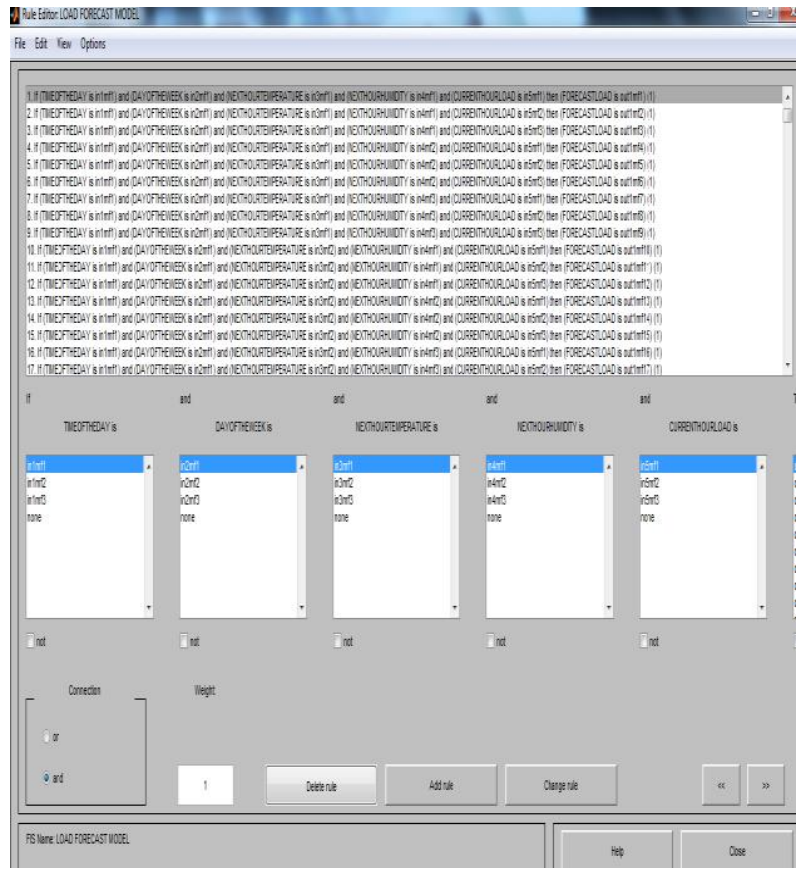

Fig 2: The dialog box for the rule editor 


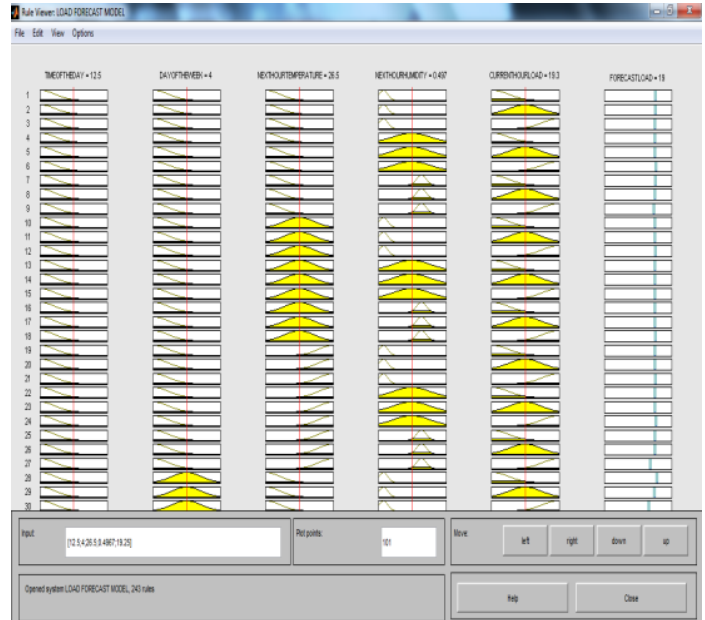

Fig 3: The dialog box of the rule viewer

\subsection{Examination of the reliability of the ANFIS-based Model:}

The design of a model serves as an end to a means, yet the performance of such model is measured by the reliability of the forecasted output. One of the major tests of reliability of a good model is that the training set performance and that of the test set are fairly similar. The key indicator of such model is the performance of the model when subjected to the standard procedural and statistical tests such as the correlation coefficient r-value, mean absolute percentage error (MAPE). For the ANFIS-Based model, using the MATLAB toolbox, the forecasted outputs are evaluated using the command line shown below.

- Calling the ANFIS Editor with the name 'LOADFORECAST' using the command anfisedit ('LOADFORECAST')

- $\quad$ Read the model using the next command:

$\mathrm{A}=$ readfis ('model name')

- Evaluate the output using the command:

$\mathrm{aa}=$ evalfis ([input 1 , input 2, input 3 . input5], a).

From the above command line, the forecasted output values will be obtained and thus fit in to the specified procedural and statistical measures defined. In order to achieve this, procedural and statistical measures such as the Mean absolute percentage error (MAPE) and Correlation Coefficient statistics using the Microsoft Excel Statistical package will be employed. The Mean Absolute Percentage Error (MAPE) computed by equation 3.2 below to evaluate the ANFIS method. MAPE is the number of test output by the difference between net output and test output divided by test output as shown. MAPE error is one of the main criteria describing the forecast method accuracy level. Its value interpretation is like 10 per cent - great accuracy; 10-20 per cent - good accuracy; 20-50 per cent - sufficient accuracy; 50 per cent and more insufficient accuracy.

MAPE $=\frac{1}{N} \sum_{M=1}^{N}$ abs $\left(\frac{\text { Load }_{\text {forecast }}(m)-\text { Load }_{\text {actual }}(m)}{\text { Load }_{\text {actual }}(m)}\right) * 100$

Where abs stands for absolute value;

$\mathrm{N}$ is the total number of hours in the testing data;

$\mathrm{m}$ stands for the $\mathrm{m}^{\text {th }}$ hour in the testing data;

$\operatorname{Load}_{\text {forecast }}(\mathrm{m})$ forecast is the forecasted load for the $\mathrm{m}^{\text {th }}$ hour.
$\operatorname{Load}_{\text {actual }}(\mathrm{m})$ actual is the actual load for the $\mathrm{m}^{\text {th }}$ hour. Similarly, Pearson Correlation Coefficient (r) is a measure of linear relationship of two variables and it ranges from -1.0 to +1.0 . Where the $r$ - value is -1.0 , it is an indication of a Perfect but negative correlation; 0 implies absence of correlation, while +1.0 is an indication of a positive and perfect correlation. Thus, using equation:

$r=\frac{\sum_{i=1}^{n}\left(A-A^{\prime}\right)\left(F-F^{\prime}\right)}{\sqrt{\sum_{i=1}^{n}\left(A-A^{\prime}\right)^{2}\left(F-F^{\prime}\right)^{2}}}$

Where, $\mathrm{r}$ is the correlation coefficient, $\mathrm{A}$ is the actual output, $\mathrm{A}^{\prime}$ is the mean of the actual output and $\mathrm{F}$ is the forecasted output and $\mathrm{F}^{\prime}$ is the mean of the forecasted output.

\section{RESULTS AND DISCUSSION}

\subsection{Simulation with Test Data}

After the ANFIS network training is completed, next hour load forecast for the upcoming hours can be calculated from the generated FIS. For example, if we want to forecast the load at 8AM of January $14^{\text {th }}, 2012$ (Jan/14/2012/8AM). One set of inputs is given as follows:

Temperature $=\mathrm{T}_{\mathrm{jan} / 14 / 2012 / 8 \mathrm{am}}$

Humidity $=\mathrm{H}_{\mathrm{jan} / 14 / 2012 / 8 \mathrm{am}}$

Day of the week $=$ Saturday (day 6 of the week)

Time of the day $=$ TOD $8 \mathrm{am}$

Current- hour load $=\mathrm{CL}_{7 \mathrm{am}}$

Using the concept above, the next hour load (forecast load) for the overall testing data of 2012 and also for some selected days were obtained. From the obtained result the mean absolute percentage error (MAPE) and the r-value (Pearson's correlation) on the overall test data and also on the selected days were also obtained as shown in table 1 to 3 .

Table 1: Actual and Forecast Load for a typical Monday

$\begin{array}{lccc}\text { Hours } & \text { Actual Load } & \text { ANFIS Forecast } & \text { APE } \\ 1 & 5.00 & 4.35 & 13.00 \\ 2 & 5.00 & 5.15 & 3.00 \\ 3 & 5.00 & 5.23 & 4.60 \\ 4 & 5.00 & 5.34 & 6.80 \\ 5 & 5.00 & 5.47 & 9.40 \\ 6 & 5.50 & 5.60 & 1.82 \\ 7 & 6.50 & 6.19 & 4.77 \\ 8 & 7.00 & 7.02 & 0.29 \\ 9 & 6.50 & 7.37 & 0.15 \\ 10 & 7.00 & 6.74 & 13.38 \\ 11 & 5.00 & 7.19 & 43.80 \\ 12 & 9.00 & 5.69 & 36.78 \\ 13 & 19.00 & 9.46 & 50.21 . \\ 14 & 20.00 & 17.64 & 11.80 \\ 15 & 21.00 & 18.29 & 12.90 \\ 16 & 23.00 & 18.96 & 19.23 \\ 17 & 23.00 & 20.87 & 9.26\end{array}$




\begin{tabular}{llcc}
18 & 22.00 & 21.03 & 4.41 \\
19 & 21.00 & 19.76 & 5.90 \\
20 & 21.00 & 18.64 & 11.20 \\
21 & 19.00 & 18.31 & 3.63 \\
22 & 18.00 & 16.26 & 9.67 \\
23 & 18.50 & 15.17 & 18.00 \\
24 & 14.00 & 15.20 & 8.57 \\
& \multicolumn{3}{c}{ MAPE $=12.61 \%$ r-Value $=0.9530$}
\end{tabular}

$\begin{array}{lccc}3 & 4.00 & 4.43 & 10.75 \\ 4 & 4.00 & 4.59 & 14.75 \\ 5 & 5.00 & 4.72 & 5.60 \\ 6 & 5.00 & 5.58 & 11.60 \\ 7 & 5.50 & 5.64 & 2.55 \\ 8 & 6.00 & 6.14 & 2.33 \\ 9 & 6.00 & 6.64 & 10.67 \\ 10 & 7.00 & 6.70 & 4.29 \\ 11 & 6.50 & 7.60 & 16.92 \\ 12 & 7.00 & 7.16 & 2.29 \\ 13 & 8.00 & 7.61 & 4.88 \\ 14 & 26.00 & 23.21 & 10.73 \\ 15 & 21.00 & 23.03 & 9.67 \\ 16 & 26.00 & 19.26 & 25.92 \\ 17 & 26.00 & 22.87 & 12.04 \\ 18 & 20.00 & 22.89 & 14.45 \\ 19 & 22.00 & 18.18 & 17.36 \\ 20 & 17.00 & 19.48 & 14.59 \\ 21 & 13.00 & 15.62 & 20.15 \\ 22 & 15.00 & 12.69 & 15.40 \\ 23 & 14.00 & 13.59 & 2.93 \\ 24 & 8.00 & 12.28 & 53.00\end{array}$

Table 2: Actual and Forecast Load for typical Wednesday

$\begin{array}{lcccc}\text { Hours } & \text { Actual Load } & \text { ANFIS Forecast } & \text { APE } & 12 \\ 1 & 10.00 & 10.11 & 1.10 & 13 \\ 2 & 4.00 & 3.89 & 2.75 & 14 \\ 3 & 3.50 & 4.01 & 14.57 & 15 \\ 4 & 4.50 & 3.68 & 18.22 & 16 \\ 5 & 4.80 & 4.65 & 3.13 & 17 \\ 6 & 5.00 & 5.18 & 3.60 & 18 \\ 7 & 5.00 & 5.73 & 14.60 & 19 \\ 8 & 7.00 & 5.69 & 18.71 & 20 \\ 9 & 6.00 & 7.48 & 24.67 & 21 \\ 10 & 6.00 & 6.42 & 7.00 & 22 \\ 11 & 5.50 & 6.38 & 16.00 & 23 \\ 12 & 6.00 & 5.96 & 0.67 & 24\end{array}$

$\begin{array}{cccc}13 & 6.00 & 6.55 & \\ 14 & 25.00 & 17.78 & 28.88\end{array}$

$\begin{array}{llll}15 & 18.00 & 21.77 & 20.94\end{array}$

$\begin{array}{llll}16 & 19.00 & 17.26 & 9.16\end{array}$

$\begin{array}{llll}17 & 21.00 & 18.08 & 13.90\end{array}$

$\begin{array}{llll}18 & 17.00 & 18.97 & 11.59\end{array}$

$\begin{array}{llll}19 & 27.70 & 16.47 & 40.54\end{array}$

$\begin{array}{llll}20 & 21.00 & 20.46 & 2.57\end{array}$

$\begin{array}{llll}21 & 16.00 & 17.80 & 11.25\end{array}$

$\begin{array}{llll}22 & 17.00 & 15.17 & 10.76\end{array}$

$\begin{array}{llll}23 & 18.00 & 15.57 & 13.50\end{array}$

$\begin{array}{llll}24 & 17.00 & 15.92 & 6.35\end{array}$

$$
\begin{aligned}
& \text { MAPE }=12.76 \% \\
& \text { r-Value }=0.9221
\end{aligned}
$$

Table 3: Actual and Forecast Load for a typical Saturday

$\begin{array}{lccc}\text { Hours } & \text { Actual Load } & \text { ANFIS Forecast } & \text { APE } \\ 1 & 14.00 & 13.98 & 0.14 \\ 2 & 4.00 & 4.31 & 7.75\end{array}$

The graph of Figures 4 to 6 shows the plot of the actual load versus ANFIS load for values obtained in tables 1 to 3 .

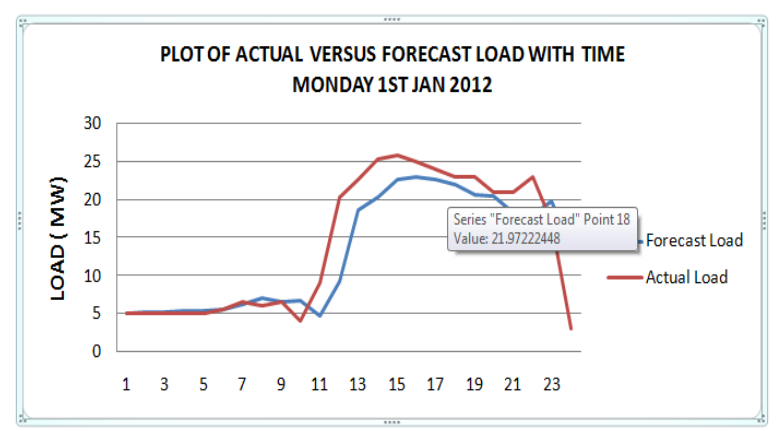

Fig 4: Actual versus forecast Load for a typical Monday

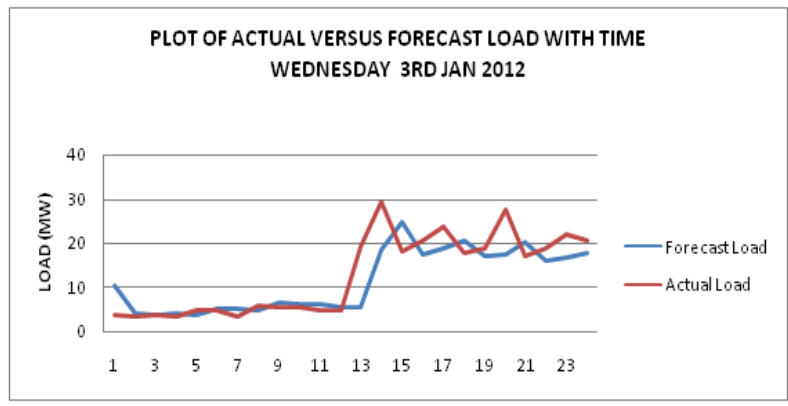

Fig5: Actual versus forecast Load for a typical Wednesday 


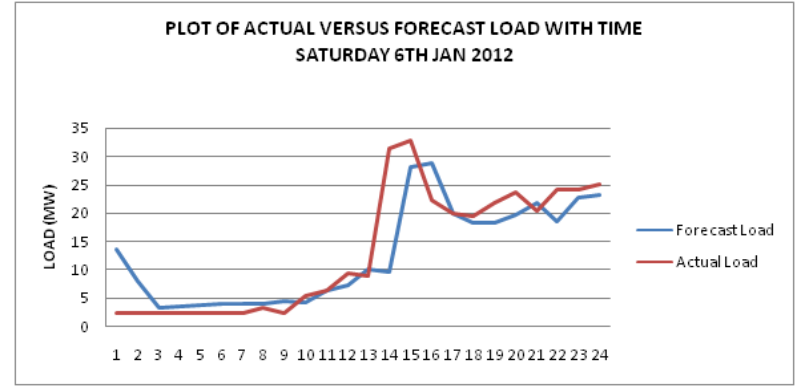

Fig 6: Actual versus forecast Load for a typical Saturday

The 3-D surface plot (control surface generated by the designed ANFIS model) shown in fig 7 and 8 depicts the relationship between certain inputs and output obtained by the designed ANFIS system. Fig 7 illustrates the relationship between the two (2) inputs temperature and day of the week, and the output which is the next hour load (forecast load). In this figure, we can see that there is a pattern such as; when the temperature is extreme, either too cold or too hot, the load would be high. Load tends to be high from Mondays and later drops when it is midweek, before rising again when it is weekend. From Figure 8, which shows the relationship between next hour temperature and time of the day with forecast load as output, it can be seen that the load tends to be high between the hours of 0 to 11 .

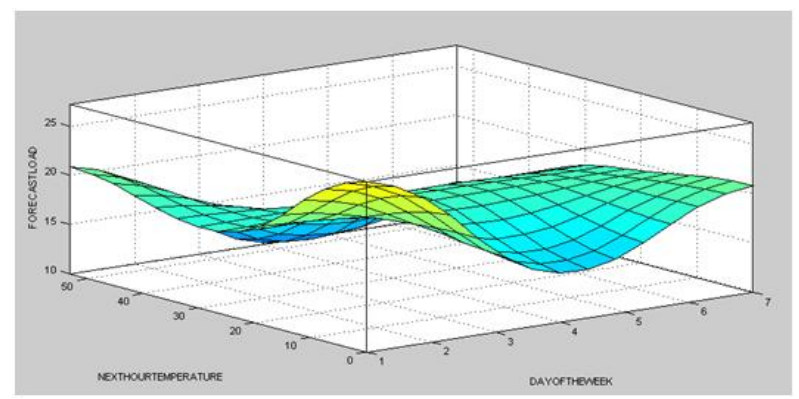

Fig 7: The relationship between temperature, day of the week, and forecasted load.

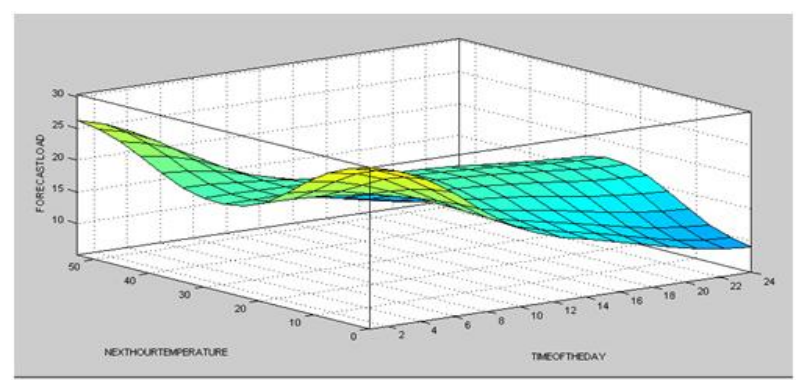

Fig 8: The relationship between temperature, hours of the day, and forecasted load.

\subsection{Discussion of the Results}

From the figures 4 to 6 , it can be seen that the difference between the actual and the forecasted load is quite small. From the above matlab command line presented in 3.5, the forecasted load values in table 1 to 3 were obtained. However, the result shown in table 1 to 3 was analyzed in order to check the accuracy of the model. The statistical measures that were employed for these analyses are the absolute percentage error (APE) and the mean absolute percentage error (MAPE) for a typical Monday (weekday), Wednesday (mid-week) Saturday (week-end) of the days in a week. However the Pearson correlation coefficient ( $\mathrm{r}$ value) using the Microsoft excel statistical package was also calculated for a typical Monday, Wednesday and Saturday. The Pearson correlation value of +0.8464 was also obtained on the overall test data. From the analysis, it was observed that the obtained mean absolute percentage value (MAPE) of $12.61 \%, 12.65 \%$ and $12.24 \%$ respectively for the ANFIS-based model using the guassian membership function for a typical Monday, Wednesday and Saturday, was in accordance to what is obtainable in most literatures. Similarly the MAPE error of $24.76 \%$ on the overall test data shows that the result obtained is sufficiently accurate.

From the r- value obtained, it is conspicuously evident that given all the assumption on the data, the ANFIS-based model will be appropriate for forecasting Maiduguri electric load demand for future planning, development and management.

\section{CONCLUSION}

The sustainable development of any region is of paramount importance to the planning, development and management of its electricity load. As such, designing a model to effectively predict the next hour load is quite apt. In this research work, ANFIS based model was designed to forecast the next hour load of Maiduguri depending on available variables (Time of the day, day of the week, temperature, humidity and load). The ANFIS based model was designed in the MATLAB ${ }^{\circledR}$ V12. (R2012b) environment. Hence based on the results obtained from this research, it can be concluded that the ANFIS based model is a better modeling tool for predicting next hour load considering the strong, high and positive correlation coefficient the model displayed.

The training and testing of the model were conducted offline, and the hourly load data used was collected from one grid. The model needs to be tested on data set from other grids, so that utility of the model can be verified for other load patterns.

The results were obtained from simulations and experimentations. No mathematical justifications could be provided for the same. Further studies on this work can incorporate additional information such as season of the year and other parameters like wind speed, sky cover and rainfall e.t.c into the network so as to obtain a more representative forecast of future load.

\section{ACKNOWLEDGMENTS}

Our sincere gratitude goes to Power holding company of Nigeria (PHCN) Maiduguri, for providing the required data.

\section{REFERENCES}

[1] Amjady, N. (2001), "Short-term hourly load forecasting using time-series modeling with peak load estimation capability", IEEE Transactions on Power Systems, 2001, v.16 (3), 498-505.

[2] Chandra, S.H, Mounika, V., and Vandana, G. (2009) "Neuro-fuzzy control in load forecasting of power sector" international journal of power system operation and energy management vol 2 pp $(70-74)$

[3] Syed-Ahmad, N., Bensenouci, A., and Abedl-Ghany, A. M., "Short Term Load Forecasting Using Artificial Neural Networks" Application to Aleppo Load Demand". Aleppo Research magazine, Oct. 2000.

[4] Nguyen, T. (2001). "Short term load forecasting Based on Adaptive Neuro-Fuzzy inference system", Journal of computers, vol 6, No 11, PP (2267-2271) 
[5] Roger-Jang, J.S. (1993). "ANFIS: Adaptive-NetworkBased Fuzzy Inference System", IEEE Trans. on Systems, Man, and Cybernetics, Vol. 23, No.3, pp. 665-685.

[6] "Load Forecasting" Chapter 12, E.A. Feinberg and Dora Genethlio, Page $269-285$, from links: www.ams.sunysb.edu and www.usda.gov

[7] Heineman, G.T., Nordman, D.A., and Plant, E.C., "The relationship between summer weather and summer loads - $\quad$ a regression analysis", IEEE Trans. Power Apparatus and systems, vol. PAS - 85, N0. 11, 1966, PP. 1144 - 1154.

[8] Corpening, S.L., Reppen, N.D., and Ringlee, R.J. " Experience with weather sensitive load models for short - $\quad$ and long- term forecasting", IEEE Trans. power Analysis and systems, vol. PAS - 92, N0.6 1973, PP. 1966 - 1972.

[9] Engle, R.F., Mustafa, C., and Rice J., "Modeling peak Electricity Demand", Journal of Forecasting, Vol 11, no.3, PP. 241-251, 1992.

[10] Hyde, O., and Hodnett, P.F., "An adaptable automated procedure for short-term electricity load forecasting," IEEE Transactions on Power Systems", vol. 12, pp. 8494, 1997.

[11] Ruzic, S., Vuckovic, A., and Nikolic, N., "Weather sensitive method for short term load forecasting in Electric Power Utility of Serbia," IEEE Transactions on Power Systems, vol. 18, pp. 1581-1586, 2003.

[12] Fan, S., and Hyndman, R.J., (2012). Short-Term Load Forecasting Based on a Semi-Parametric Additive Model. IEEE Transactions on Power Systems, 27(1), 134-141
[13] Alfares, H.K., Nazeeruddin, M., (2002). Electric Load Forecasting: Literature Survey and Classification of Methods. International Journal of Systems Science, Vol. 33, No. 1, pp 23-34

[14] Islam, B.U. (2011). Comparison of Conventional and Modern Load Forecasting Techniques based on Artificial Intelligence and Expert Systems. International Journal of Computer Science Issues, Vol. 8, Issue 5, No 3, pp 504-513 Lefteri H. Tsoukalas, Robert. Uhrig, 1997 - Fuzzy and Neural Approaches in Engineering, Wiley Interscience.

[15] Desouky, A.A., and Elkateb, M.M., (2000). Hybrid Adaptive Techniques for Electric-Load Forecast using ANN and ARIMA. IEE Proceedings of Generation, Transmission and Distribution. 147(4), 213 - 217.

[16] Bakirtzis, A. G., Theocharis, J. B., Kiartzis, S. J., and Satsios, K. J., " Short term load forecasting using fuzzy neural networks," IEEE Transactions on Power Systems, vol. 10, pp. 1518-1524, 1995.

[17] Papadakis, S. E., Theocharis, J. B., Kiartzis, S. J., and Bakirtzis, A. G., "A novel approach to shortterm load forecasting using fuzzy neural networks," IEEE Transactions on Power Systems, vol. 13, pp. 480-492, 1998.

[18] Dash, P. K., Liew, A. C., and Rahman, S., "Fuzzy neural network and fuzzy expert system for load forecasting," IEE Proceedings - Generation, Transmission and Distribution, vol. 143, pp. 106-114, 1996.

[19] Srinivasan, D., Tan, S. S., Chang, C. S., and Chan, E. K., "Practical implementation of a hybrid fuzzy neural network for one-day-ahead load forecasting," IEE Proceedings - Generation, Transmission and Distribution, vol. 145, pp. 687-692, 1998 